\begin{tabular}{|l|c|c|c|c|c|}
\hline Acuity level & $\mathbf{1}$ & $\mathbf{2}$ & $\mathbf{3}$ & $\mathbf{4}$ & $\mathbf{5}$ \\
\hline Triage color & Red & Orange & Yellow & Green & Blue \\
\hline $\begin{array}{c}\text { Recommended } \\
\text { wait time }\end{array}$ & $0 \mathrm{~min}$ & $15 \mathrm{~min}$ & 1.5 hour & 4 hours & 12 hours \\
\hline $\begin{array}{c}\text { Was the } \\
\text { recommended } \\
\text { time exceeded? }\end{array}$ & No & No & Yes & Yes & Yes \\
\hline Did death occure? & Yes & Yes & Yes & No & No \\
\hline Visits by acuity level & $0.56 \%$ & $2.57 \%$ & $17.84 \%$ & $73.29 \%$ & $5.74 \%$ \\
\hline $\begin{array}{c}\text { Hospitalization by } \\
\text { acuity level }\end{array}$ & $100 \%$ & $76.49 \%$ & $43.1 \%$ & $14.35 \%$ & $2.08 \%$ \\
\hline
\end{tabular}

Table 1. The ESI modification in practice.

Prehosp Disaster Med 2017;32(Suppl. 1):s238-s239

doi:10.1017/S1049023X17006112

The Relevant Factors of the Early Prognosis and the Need of Intensive Medical Resources of Patients with Multiple Injuries Di Hao

Department Of Emergency, First Affiliated Hospital of Sichuan University, Chengdu/China

Study/Objective: To estimate the early prognosis and evaluate the need of intensive medical resources of patients with multiple injuries.

Background: A large amount of research and clinical practice indicates that the multiple injuries are urgent and the illness change of a patient's condition is rapid, which leads to the a high mortality rate. We can take some early and effective methods of triage to make patients receive timely, effective treatment, thus to reduce the mortality rate. In that case, we need some early and effective indicators of triage.

Methods: We recruited 115 patients with multiple injuries admitted to emergency department of West China Hospital, Sichuan University between March 2016 and May 2016 and collected 19 clinical indicators from each patient. The indicators included gender, age, temperature, heart rate, respiratory rate, peripheral oxygen saturation, systolic pressure, diastolic pressure, power of hydrogen $(\mathrm{PH})$, hemoglobin, base excess $(\mathrm{BE})$, serum potassium, serum sodium, serum calcium, lactic acid, glucose, partial pressure of oxygen (PO2), carbon dioxide partial pressure (PCO2), and peritoneal effusion. We analyzed the correlation of these indicators with deaths within the first 24 hours, emergency surgery, admissions to intensive care unit (ICU), and length of ICU stay through the method of a rank sum test and logistic regression with SPSS 19.0.

Results: The results showed that the possibility of death (A) could be expressed as: $\mathrm{A}=-0.276 * \mathrm{BE}(\mathrm{mmol}),-3.005^{*} \mathrm{~T}\left({ }^{\circ} \mathrm{C}\right)$ $0.073^{*} \mathrm{PO} 2(\mathrm{mmHg}) 110.843$ and the need of admissions to intensive care unit $(\mathrm{B})$ as: $\mathrm{B}=1.007^{*}$ peritoneal effusion + $0.140^{*}$ glucose $(\mathrm{mmol} / \mathrm{L})-3.224$.

Conclusion: BE, T, PO2 may be useful in early forecasting the prognosis of patients with multiple injuries; glucose and peritoneal effusion can evaluate if the patient needs the intensive medical resources.

Prehosp Disaster Med 2017;32(Suppl. 1):s239

doi:10.1017/S1049023X17006124
Validation of CRISTO as a Triage Tool in Emergencies and Disasters

Pablo Jiménez ${ }^{1}$, Gessela Carvajal ${ }^{2}$, Xavier Betancourt ${ }^{1}$, Patricia Mogrovejo ${ }^{1}$, Nicole A. Jiménez ${ }^{2}$

1. Facultad De Ciencias De La Salud Eugenio Espejo, Universidad Tecnologica Equinoccial, Quito/Ecuador

2. Corporación SAVINMED, Quito/Ecuador

Study/Objective: Our objective was to validate CRISTO (C: Walking; R: Respiratory failure; I: Unconscious/neurological

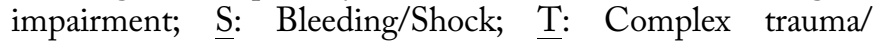
Behavioral disorder; O: Others), as a method to be applied in victim classification, as well as in the comparison of efficiency and execution time regarding the Standardized Testing and Reporting (STAR) method.

Background: In April 2016, Ecuador suffered an earthquake which caused 671 deaths and left 8,690 people homeless. This event tested the capacity of response and the implementation of protocols, including triage in the country. START is a validated and widely used method for victim classification; however, the average evaluation time it has, among other things, has made us question its effectiveness in major disasters like this one.

Methods: This is a descriptive and comparative study of two triage methods. A total of 12 simulated patients were evaluated by 10 First Response Teams during a disaster simulation exercise; five for each triage method, selected by drawing lots. Triage was carried out by Technologists in Medical Emergencies, or Medical Doctors with training in both methods and previous experience in each procedure. The simulation patients were 1 black, 4 red, 3 yellow and 4 green. We compared sorting efficiency and evaluation time for each method.

Results: The percentage of positive answers with CRISTO was $85 \%$, and with START was $73.3 \%(p=0.21)$; nevertheless, when we evaluated triage time, CRISTO $(10.8 \mathrm{sec}$.) was faster than START (9.5 sec.), $\mathrm{p}=0.025$.

Conclusion: In conclusion, CRISTO is a reliable and fast method of triage, ensuring greater patient care during large events with multiple victims.

Prehosp Disaster Med 2017;32(Suppl. 1):s239

doi:10.1017/S1049023X17006136

\section{The Use of the Mobile Information and Communication Technologies in Mass-Casualty Incident and Disaster Management - A Medical Triage System \\ Arkadiusz Trzos}

Department Of Disaster And Emergency Medicine, Chair Of Anaesthesiology And Intensive Care, Jagiellonian University Medical College, Cracow/Poland

Study/Objective: Worldwide, Mobile Information and Communication Technologies (ICT) have been used in prehospital emergency care and emergency and disaster medicine. In Poland, the use of ICT in routine emergency practice does not raise any concerns, but special application used in mass-casualty incidents and disasters is still being discussed.

Background: The development of "intelligent" Command Support System (CSS) for Emergency and Disaster Medicine is the aim of this study. The problem of the correct allocation of 